Article

\title{
Gain and Threshold Current in Type II In(As)Sb Mid-Infrared Quantum Dot Lasers
}

\section{Qi Lu *, Qiandong Zhuang and Anthony Krier}

Physics Department, Lancaster University, Lancaster LA1 4YB, UK;

E-Mails: q.zhuang@lancaster.ac.uk (Q.Z.); a.krier@lancaster.ac.uk (A.K.)

* Author to whom correspondence should be addressed; E-Mail: q.lu3@lancaster.ac.uk;

Tel.: +44-015-24-597-50; Fax: +44-015-24-593-651.

Received: 27 February 2015 / Accepted: 11 April 2015 / Published: 15 April 2015

\begin{abstract}
In this work, we improved the performance of mid-infrared type II InSb/InAs quantum dot (QD) laser diodes by incorporating a lattice-matched p-InAsSbP cladding layer. The resulting devices exhibited emission around $3.1 \mu \mathrm{m}$ and operated up to $120 \mathrm{~K}$ in pulsed mode, which is the highest working temperature for this type of QD laser. The modal gain was estimated to be $2.9 \mathrm{~cm}^{-1}$ per QD layer. A large blue shift $(\sim 150 \mathrm{~nm})$ was observed in the spontaneous emission spectrum below threshold due to charging effects. Because of the QD size distribution, only a small fraction of QDs achieve threshold at the same injection level at $4 \mathrm{~K}$. Carrier leakage from the waveguide into the cladding layers was found to be the main reason for the high threshold current at higher temperatures.
\end{abstract}

Keywords: quantum dots; mid-infrared; semiconductor lasers

\section{Introduction}

The mid-infrared wavelength range $(2-5 \mu \mathrm{m})$ continues to attract significant research interest due to its importance for various potential applications, such as optical gas sensing, environmental pollution monitoring, chemical process control, non-invasive medical diagnosis, tunable IR spectroscopy, laser surgery and infrared countermeasures [1]. Efficient and cost-effective solid-state laser sources emitting in this spectral range are required in many applications. In recent years, mid-infrared quantum well (QW) lasers have made significant progresses in reducing the threshold current density, increasing maximum working temperature and output power [2,3]. Moreover, the quantum cascade lasers (QCLs) and inter-band cascade lasers (ICLs), which make use of the inter sub-band transition, have achieved 
superior performance [4,5]. However, these types of devices suffer from lower wall plug efficiency and higher turn-on voltages than QW lasers. In addition, QCLs and ICLs are typically composed of hundreds of thin layers, making their growth more challenging. From theoretical studies, due to their discrete density of states, QDs have higher material gain, narrower gain spectrum, and are less temperature sensitive than QWs [6], making them the ideal candidate as the gain medium in semiconductor lasers. Compared with QCLs and ICLs, QD lasers also have the advantage of less complicated growth. Although the growth conditions for successful realization of QDs needs careful attention to detail to obtain the correct size distribution and area density, once these conditions are established the active region growth can be easily completed and does not require an extended growth period. Despite these potential merits, to date there are still very few reports on QD lasers in the mid-infrared range and their performance lags behind the aforementioned types of lasers. However, type II InSb/InAs QDs grown by molecular beam epitaxy (MBE), which have exhibited room temperature mid-infrared photo-luminescence (PL) [7] and electro-luminescence (EL) [8], could be a route towards QD light sources and lasers emitting beyond $3 \mu \mathrm{m}$.

In this work, we present broad area InSb QD laser diodes grown on InAs substrates, which emitted around $3.1 \mu \mathrm{m}$. The structure incorporated a $\mathrm{p}-\mathrm{InAs} \mathrm{s}_{0.61} \mathrm{Sb}_{0.13} \mathrm{P}_{0.26}$ cladding layer grown by liquid phase epitaxy (LPE). The laser diodes worked up to $120 \mathrm{~K}$ in pulsed mode, which is a significant improvement compared with previous work $[9,10]$. The gain of these type II QDs was estimated and the different contributions to the threshold current were studied.

\section{Experimental Section}

The QD lasers were grown on (100) oriented p-InAs substrates. Firstly, a $2 \mu \mathrm{m}$ thick InAs0.61 $\mathrm{Sb}_{0.13} \mathrm{P}_{0.26}$ cladding layer was deposited by LPE from an indium-rich melt. The material was p-type doped with $\mathrm{Zn}$ to a concentration of $\sim 2 \times 10^{18} \mathrm{~cm}^{-3}$. The rest of the structure was then grown in a VG V80H MBE reactor. Ten layers of InSb QDs with $20 \mathrm{~nm}$ thick InAs spacers between each layer were deposited in the middle of a $1.2 \mu \mathrm{m}$ thick undoped $\left(1 \times 10^{16} \mathrm{~cm}^{-3}\right)$ InAs waveguide region. On top of that, an n-doped cladding layer was grown, which was composed of $0.3 \mu \mathrm{m}$ thick n-InAs with a doping concentration of $1 \times 10^{17} \mathrm{~cm}^{-3}$ and $1.7 \mu \mathrm{m}$ thick $\mathrm{n}^{+}$-InAs with a doping concentration of $5 \times 10^{18} \mathrm{~cm}^{-3}$ which had a lower refractive index to complete the waveguide. The structure and band alignment of the resulting lasers are sketched in Figure 1a.

Current-voltage measurements from the fabricated lasers revealed good $\mathrm{p}-\mathrm{i}-\mathrm{n}$ diode rectifying characteristics. As shown in Figure $1 \mathrm{~b}$, the turn-on voltage decreased from $0.34 \mathrm{~V}$ at $4 \mathrm{~K}$ to $0.10 \mathrm{~V}$ at $300 \mathrm{~K}$. The diode series resistance was $\sim 2 \Omega$ for all temperatures and the reverse leakage current was $4 \mathrm{~mA}(0.5 \mathrm{~V}$ reverse bias) at $300 \mathrm{~K}$.

Unlike the common Stranski-Krastanov growth of QDs, which mainly relies on the strain effect between the deposited material and the substrate [11], in this case the growth of the InSb QDs is based on the As-to-Sb anion exchange reaction, which is achieved by exposing the substrate to $\mathrm{Sb}_{2}$ flux for a very short time interval. The substrate temperature during dot formation can be adjusted in the interval $320-450{ }^{\circ} \mathrm{C}$, resulting in InSb layers of thickness in the range 0.5-0.9 monolayer (ML). Ideally, larger QDs are required for use in lasers to maintain sufficient hole localization. For QDs grown using the exchange reaction, this is only possible at growth temperatures below $350{ }^{\circ} \mathrm{C}$. However, it was found 
that at growth temperatures in the range $320-350{ }^{\circ} \mathrm{C}$ the PL intensity decreased dramatically because of the poor material quality. Furthermore, strong Sb segregation can occur on the InAs surface forming rougher interfaces. Thus, to avoid this problem, in our laser structure the QDs were grown at higher substrate temperature $\left(420{ }^{\circ} \mathrm{C}\right)$. Then to increase the size of the QDs, InSb was deposited for $4 \mathrm{~s}$ following the $\mathrm{Sb}$ exchange reaction, resulting in QDs about $0.8 \mathrm{ML}$ in thickness (i.e., a few nanometers in each dimension) but with a very high density $\left(\sim 10^{12} \mathrm{~cm}^{-2}\right)$. More details of the QD exchange growth can be found in our earlier work [8]. In agreement with previous reports, no wetting layer was observed. The QDs grown using this method typically emitted in the 3-4 $\mu \mathrm{m}$ spectral range, and the emission wavelength was dependent upon the substrate temperature and $\mathrm{Sb}_{2}$ flux exposure time during the growth [8,9]. After growth, broad area laser stripes were fabricated from the sample using conventional photolithography and wet chemical etching. Ti/Au $(20 \mathrm{~nm} / 200 \mathrm{~nm})$ metallization was thermally evaporated on both sides to form the ohmic contacts. The lasers were fabricated to be $75 \mu \mathrm{m}$ in width and of selected varying cavity lengths. The diodes were mounted and wire-bonded on TO-46 headers for measurements and testing.

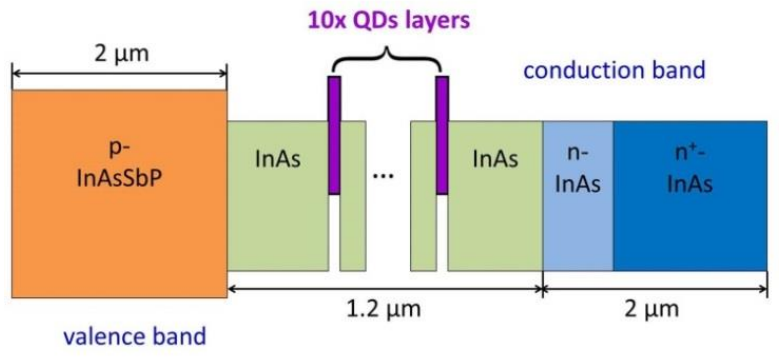

(a)

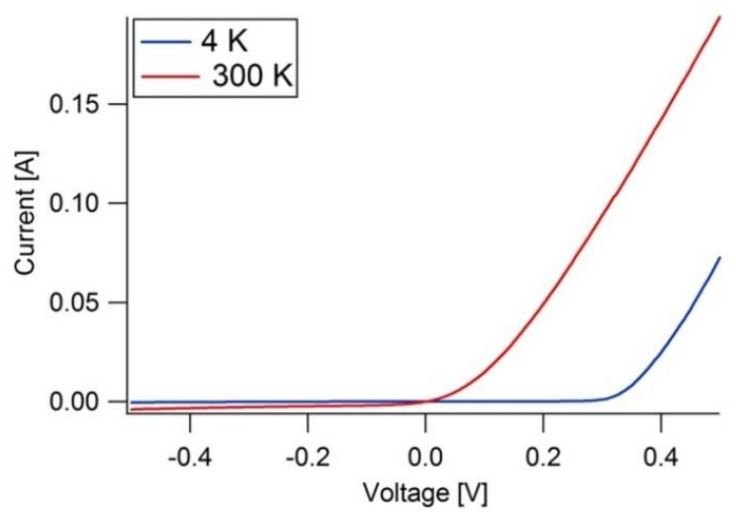

(b)

Figure 1. (a) Illustration of the QD laser structure and the conduction and valence band alignments. (b) Current-voltage curves of the laser diode measured at $4 \mathrm{~K}$ and $300 \mathrm{~K}$.

\section{Results and Discussion}

\subsection{Gain from InSb QDs}

QD laser diodes with different cavity lengths were tested in pulsed mode (100 ns pulse width, $2 \mathrm{kHz}$ repetition rate) from $4 \mathrm{~K}$ to $120 \mathrm{~K}$. The threshold current density $\left(\mathrm{J}_{\mathrm{th}}\right)$ increased from $1.59 \mathrm{kAcm}^{-2}$ to $4.68 \mathrm{kAcm}^{-2}$ as the cavity length was reduced from $1.17 \mathrm{~mm}$ to $0.33 \mathrm{~mm}$. Using the same method as for QW lasers [12], the gain of our InSb QD lasers was estimated from the relation between $\mathrm{J}_{\mathrm{th}}$ and different laser cavity lengths L, using:

$$
J_{t h}=\frac{n_{w} J_{t r}}{\eta_{i}} \exp \left(\frac{\alpha_{i}+\frac{1}{L} \ln \left(\frac{1}{R}\right)}{n_{w} \Gamma_{w} G_{0}}\right)
$$


where $n_{w}$ is the number of QD layers, $J_{t r}$ is the transparency current density per layer, $\eta_{i}$ is the internal quantum efficiency, $\alpha_{\mathrm{i}}$ is the waveguide loss, $\mathrm{R}$ is the reflection coefficient of the end facet, $\Gamma_{\mathrm{w}} \mathrm{G}_{0}$ is the modal gain in which $\Gamma_{\mathrm{w}}$ is the optical confinement factor per layer and $\mathrm{G}_{0}$ is the material gain coefficient. By making a linear fit between $\ln \left(\mathrm{J}_{\mathrm{th}}\right)$ and $1 / \mathrm{L}$, the modal gain can be extracted.

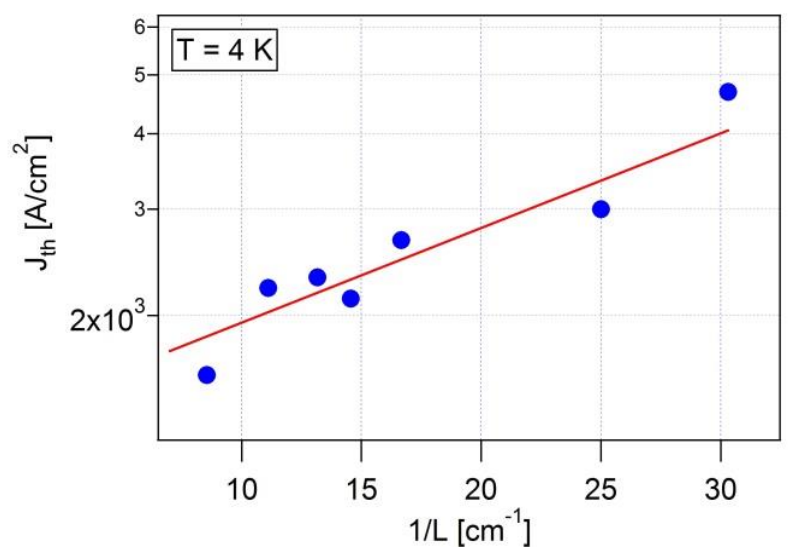

Figure 2. Threshold current density $\left(\mathrm{J}_{\mathrm{th}}\right) v s$. reciprocal cavity length $(1 / \mathrm{L})$ of the laser diodes at $4 \mathrm{~K}$. The red line shows the linear fit between $\ln \left(\mathrm{J}_{\mathrm{th}}\right)$ and $1 / \mathrm{L}$.

From Figure 2, the modal gain of the QD laser was estimated to be $\sim 29 \mathrm{~cm}^{-1}$, (i.e., $2.9 \mathrm{~cm}^{-1}$ per QD layer on average). This value is lower than typical (InAs) type I QDs emitting in the near-infrared range which is in the order of $10 \mathrm{~cm}^{-1}[13,14]$, but is close to that of type II QWs emitting at a similar wavelength [12]. The relatively lower modal gain from type II structures is closely related to the large spatial separation between electrons and holes compared with type I structures, which results in a much smaller electron-hole wave function overlap.

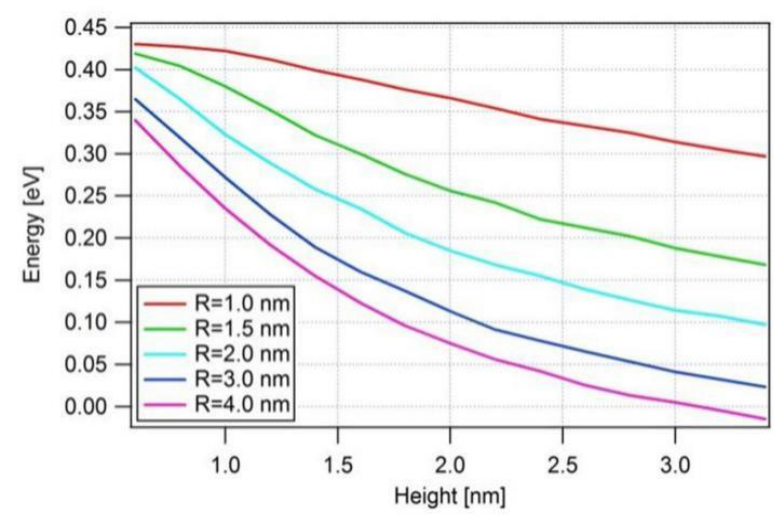

(a)

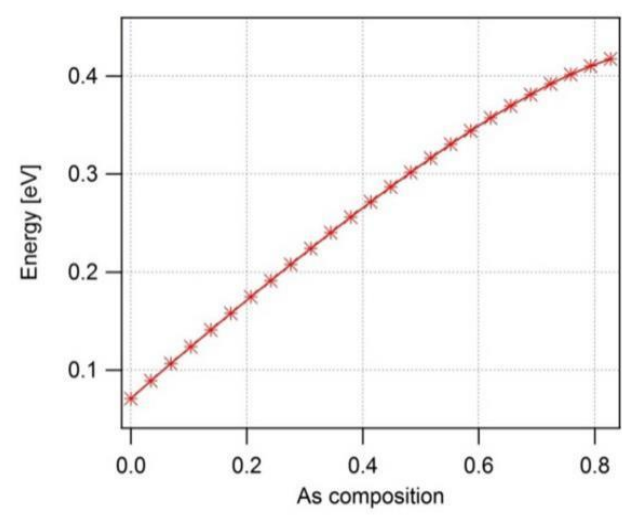

(b)

Figure 3. (a) Calculated transition energy of InSb QD as a function of the QD height for different radius $(\mathrm{R})$ values at $4 \mathrm{~K}$. (b) Calculated transition energy of $\operatorname{InAsx}_{x} \mathrm{Sb}_{1-\mathrm{x}} \mathrm{QD}$ as a function of As composition at $4 \mathrm{~K}$, where the QD size is fixed at $3 \mathrm{~nm}$ base radius and $2 \mathrm{~nm}$ height. 
From previous reports [7-9,15], the peak emission wavelength of such InSb QDs typically falls between 3.2 and $3.8 \mu \mathrm{m}$ at low temperatures, corresponding to transition energies in the range $0.326-0.388 \mathrm{eV}$. The dependence of transition energies of pure InSb QD on the radius and height of the QDs at $4 \mathrm{~K}$ were evaluated using three-dimensional single band Schrödinger calculations in COMSOL multiphysics. The results are plotted in Figure 3a, where the transition energy exhibits a strong dependence on the QD size. For example, with a fixed radius of $2 \mathrm{~nm}$, a $0.5 \mathrm{~nm}$ change in QD height (from $1.5 \mathrm{~nm}$ to $2 \mathrm{~nm}$ ) resulted in about $70 \mathrm{meV}$ difference in transition energy. Although the COMSOL calculation is a much simpler estimation than the k.p calculations, the values obtained here are in fact in good agreement with the results from $6 \times 6 \mathrm{k} . \mathrm{p}$ calculations [16]. Assuming the QD was composed of pure InSb, it was found from both k.p modeling in [16] and COMSOL calculation from Figure 3a that, to achieve typical transition energy of $0.35 \mathrm{eV}$, the QD height needed to be about $1.0 \mathrm{~nm}$ and radius 1.25-1.5 $\mathrm{nm}$. However, high resolution microscopic images reported in [17] revealed that the QDs could possibly be much larger in size ( $\sim 3 \mathrm{~nm}$ base radius and $\sim 2.5 \mathrm{~nm}$ in height). The most probable reason for this disagreement is that instead of pure InSb, the QDs may contain a large proportion of As. By fixing the QD size according to the microscopic image in [17], the transition energy dependence on the composition of As was also calculated by the same method in COMSOL and plotted in Figure 3b. To obtain the $0.35 \mathrm{eV}$ transition energy, the QD composition should be close to InAs $0.6 \mathrm{Sb}_{0.4}$. It needs to be stressed that one image cannot represent all of the QDs in the structure and it is most likely that there are deviations in both QD size and composition. Thus, both QDs with pure InSb in an extremely small size or larger QDs with more As in the composition can exist in the same sample.

In the In(As)Sb QDs, holes are the only confined carriers, while electrons can move freely in the InAs matrix. Both COMSOL simulations and $6 \times 6 \mathrm{k} . p$ calculations [16] confirm that the ground heavy hole $(\mathrm{GHH})$ state was the only confined energy state within QDs of base radius $1.25-3 \mathrm{~nm}$. For both pure InSb QDs $(\sim 1.25 \mathrm{~nm})$ and larger InAs $0.6 \mathrm{Sb}_{0.4}$ QDs $(\sim 3 \mathrm{~nm})$, the electron-hole wave function overlap lies within the range 35\%-40\% (using both simulation methods), which is lower than in type I QDs and W-structures [18,19]. However, due to the high QD density, an exceptionally high material gain of $\sim 20 \times 10^{4} \mathrm{~cm}^{-1}$ can be estimated for our type II QD, which is in the same range as for type I QDs [13]. Despite the smaller electron-hole wave function overlap, these type II QDs can serve as an efficient gain medium for mid-infrared laser diodes.

The laser diodes emitted between 3.02-3.11 $\mu \mathrm{m}$ and the details of the laser spectrum can be revealed by using high resolution Fourier transform infrared (FTIR) spectroscopy. The measured spectra of one laser diode at $4 \mathrm{~K}$ with different drive currents are plotted in Figure 4. The relatively broad overall spectra are probably caused by the size distribution of QDs. Two representative groups of modes are circled in this figure. At lower current injection, the longer wavelengths modes (circled in red) appeared first. With higher current injection, the shorter wavelengths modes (circle in blue) became stronger. This means the longer wavelength modes reach threshold first, associated with coherent emission from the larger QDs. This is also consistent with the transfer of holes between adjacent QDs by tunneling or thermal excitation [20] so that QDs with lower GHH states (i.e., emitting at longer wavelengths) become occupied first as current injection is increased. 


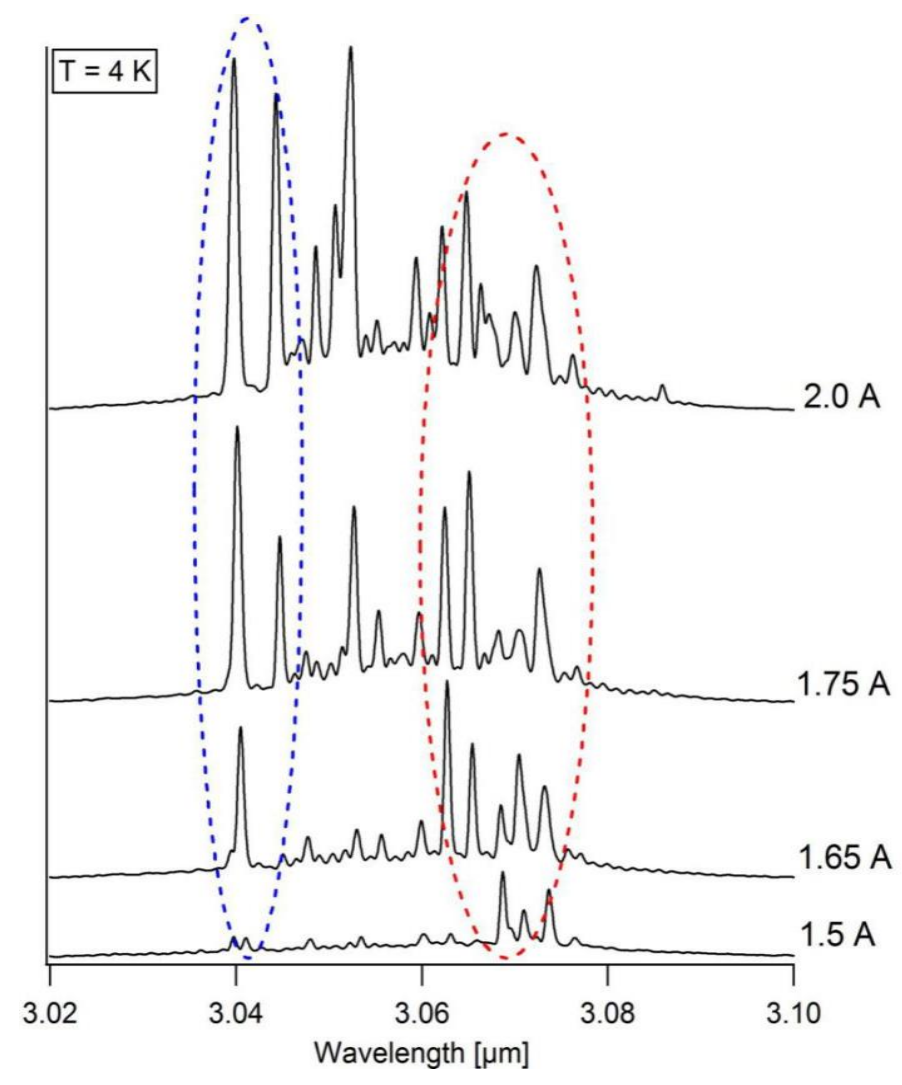

Figure 4. Multimodal laser spectra measured at $4 \mathrm{~K}$ using different pulsed drive currents. Two representative groups of modes are identified and are circled using red and blue dashed lines in the figure.

\subsection{Spontaneous Emission}

The spontaneous electro-luminescence (SE) emission spectra from the laser diodes were measured from the end facet when the injected current was below threshold. An interesting phenomenon was observed that with increasing current injection, there was a large blue shift of the SE peak position. In Figure 5a, the SE peaked at $3.236 \mu \mathrm{m}$ at $50 \mathrm{~mA}$ and moved down to $3.094 \mu \mathrm{m}$ at $750 \mathrm{~mA}$, corresponding to an energy difference of about $18 \mathrm{meV}$, but without significant broadening. The above threshold spectrum envelope at $1.0 \mathrm{~A}$ is also shown in the low resolution spectra of Figure 5a, where the lasing peak lies very close to the peak of the SE spectrum at $750 \mathrm{~mA}$. 


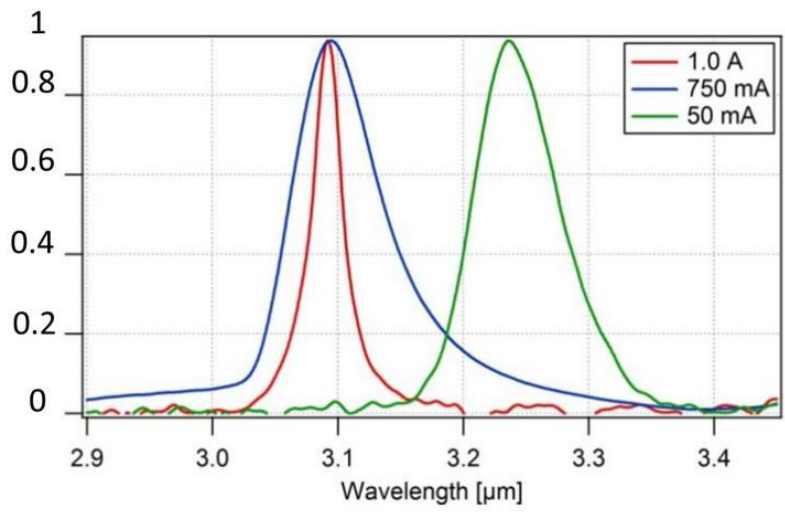

(a)

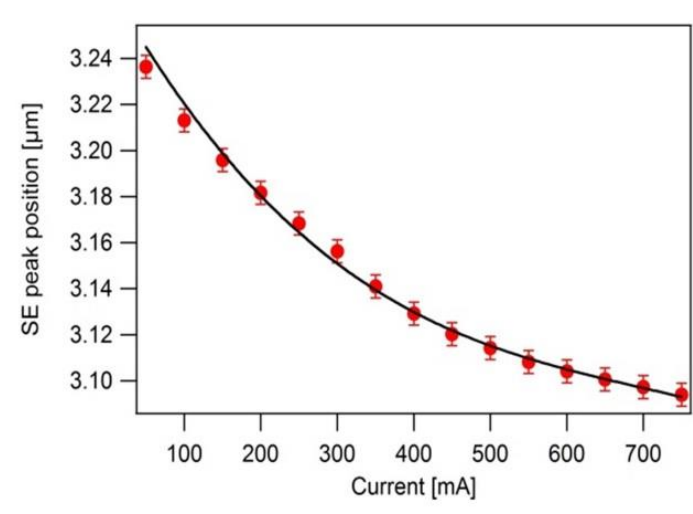

(b)

Figure 5. (a) Comparison of the sub-threshold emission spectrum at $50 \mathrm{~mA}$ current injection (green curve) and at $750 \mathrm{~mA}$ current injection (blue curve) with the laser spectrum envelope just above threshold at $1.0 \mathrm{~A}$ current injection (red curve). The peak intensities of the three spectra have been normalized. (b) Below threshold spontaneous emission peak wavelength shift with increasing current from a laser diode measured at $4 \mathrm{~K}$. The threshold current of this laser $\mathrm{I}_{\mathrm{th}}=950 \mathrm{~mA}$.

The SE peak position dependence on the injected current is shown in Figure 5b. In the lower current range the rate of the SE peak shift is greater than in the higher current range. Since there is only one quantized heavy hole state in our QDs, one possible reason to explain the SE peak shift is the band-bending effect caused by the electric field in type II structures. When the holes occupy the QDs, the electrons can be attracted closer to them, causing the conduction band to bend into a triangular shape, as illustrated in Figure 6a. Quantized electron states may occur in the triangular potential, resulting in higher recombination energy. Blue shifts caused by band-bending have already been reported from other kinds of type II QWs [21] and QDs [22]. The relation between the injected current and wavelength shift can be written as [21]:

$$
\Delta E_{B} \propto I^{1 / 3}
$$

where $\Delta \mathrm{E}_{\mathrm{B}}$ is the transition energy shift caused by band-bending, and I is the injected current.

Another possible reason that can cause a blue shift of the SE peak is the charging of QDs. In type II structures, since electrons and holes are spatially separated, the repulsive Coulomb force between carriers of the same type (the injected holes in our case) is much greater than the attractive Coulomb force between electrons and holes $[23,24]$. The strong interaction between the two holes in GHH state inside the QD can decrease the hole confinement energy, resulting in a blue shift of the SE peak. A single QD can be modelled as a cylindrical capacitor as sketched in Figure 6b. The relation between the transition energy shift arising from this charging effect and injected current can be obtained as:

$$
\Delta E_{C} \propto I^{1 / 2}
$$

where $\Delta E_{\mathrm{C}}$ is the transition energy shift caused by charging, and $I$ is the injected current. 


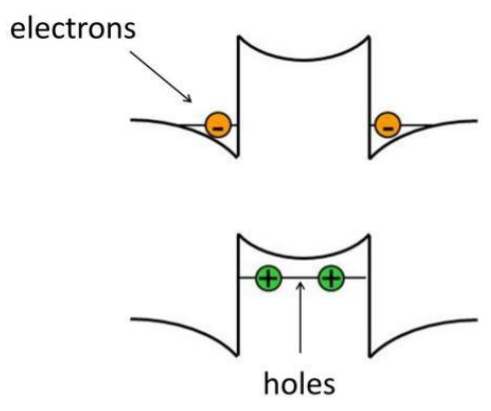

(a)

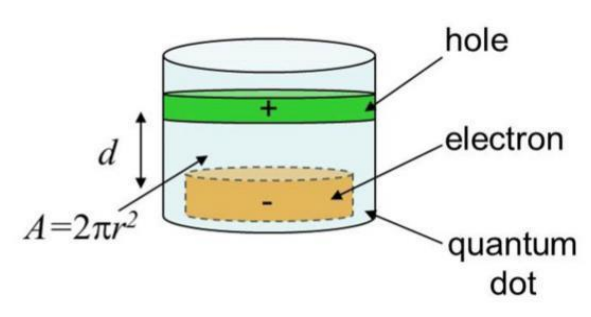

(b)

Figure 6. (a) Illustration of the band-bending effects caused by the electric field in type II nanostructures. (b) Illustration of the simple capacitor model for a QD associated with charging effects.

Comparing Equations (2) and (3), it is evident that the energy shifts caused by these two mechanisms have different dependence on the injected current. A logarithmic plot of the measured SE peak shift against the current is shown in Figure 7. The slope of the linear fit (blue line) in this plot was 0.53, which confirms that the QD charging effect is responsible for the blue shift of the SE peak.

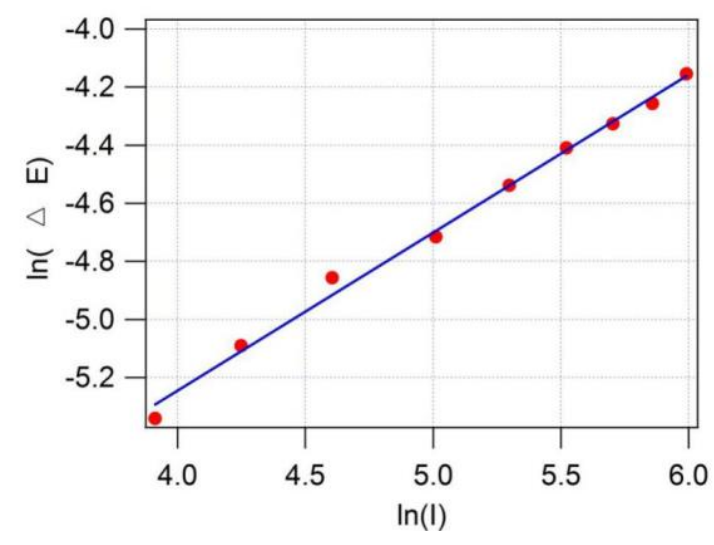

Figure 7. Logarithmic plot of the SE peak shift $(\Delta \mathrm{E})$ at different injected currents (I). The blue line is a linear fit between $\ln (\Delta \mathrm{E})$ and $\ln (\mathrm{I})$. The slope $=0.53$.

\subsection{Non-Radiative current}

With increasing temperature, the threshold current density $\mathbf{J}_{\text {th }}$ of the laser diodes increased rapidly. However, compared to previous reports the measured maximum working temperature $\left(\mathrm{T}_{\max }\right)$ was improved from $60 \mathrm{~K}$ to $120 \mathrm{~K}$, because of improved waveguide design and cladding layer material quality. The relationship between $\mathrm{J}_{\text {th }}$ and temperature was plotted in Figure 8, where the characteristic temperature, $\mathrm{T}_{0}$ was estimated to be $101 \mathrm{~K}$ when the temperature was below $50 \mathrm{~K}$. For the temperature range between $50 \mathrm{~K}$ and $120 \mathrm{~K}$, the value of $\mathrm{T}_{0}$ dropped to $48 \mathrm{~K}$. Possible contributions towards the increase of $\mathrm{J}_{\mathrm{th}}$ and decrease of $\mathrm{T}_{0}$ include: the leakage current, Auger recombination and ShockleyRead-Hall (SRH) recombination. The total current density $\mathbf{J}_{\text {tot }}$ can be expressed as [25]:

$$
J_{\text {tot }}(T)=J_{\text {leak }}+J_{\text {rad }}+J_{S H R}+J_{\text {Auger }}=J_{\text {leak }}+J_{\text {rad }}+e d_{\text {eff }}\left(A n+C n^{3}\right)
$$


where $\mathrm{J}_{\text {leak }}, \mathrm{J}_{\mathrm{rad}}, \mathrm{J}_{\mathrm{SRH}}$ and $\mathrm{J}_{\text {Auger }}$ are the leakage current, radiative current, SRH current and Auger current, respectively. The terms $\mathrm{An}$ and $\mathrm{Cn}^{3}$ represent contributions from SRH and Auger recombination, where $\mathrm{n}$ is the threshold carrier density and $\mathrm{d}_{\mathrm{eff}}$ is the effective width of the active region. The relation between temperature and $\mathrm{J}_{\text {leak }}$ was taken from [26]. In ideal QD lasers, $\mathbf{J}_{\text {rad }}$ should be temperature independent $[6,27]$. The calculated $\mathrm{J}_{\mathrm{SRH}}, \mathrm{J}_{\text {Auger }}$ and $\mathrm{J}_{\text {leak }}$ were plotted in Figure 8, where we have used representative values of the respective coefficients taken from the literature [26,28]. The $\mathrm{J}_{\text {tot }}$ (black curve) was then fitted to the experimental data $\mathrm{J}_{\exp }$ by adjusting the constant value of $\mathrm{J}_{\mathrm{rad}}$.

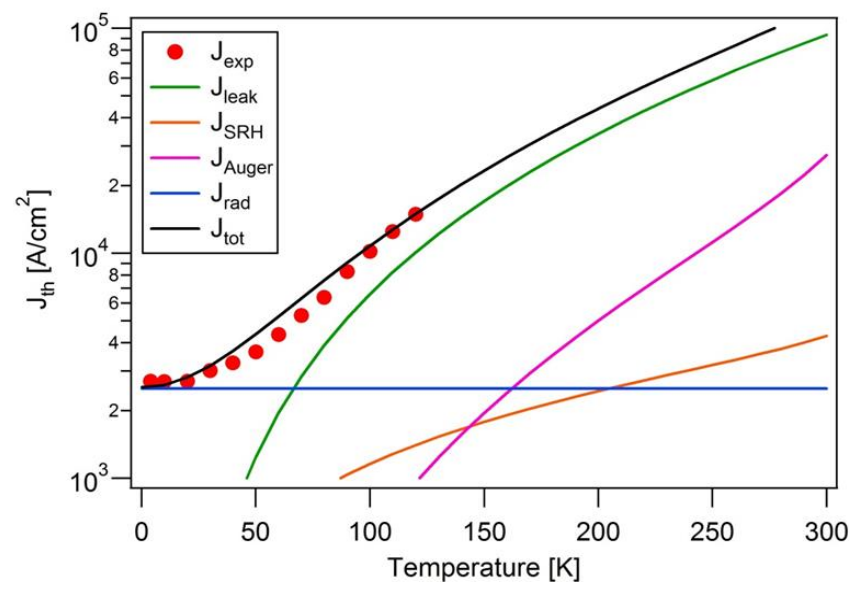

Figure 8. Measured threshold current density (red dots) of the $\mathrm{In}(\mathrm{As}) \mathrm{Sb}$ QD laser at different temperatures. Calculated $\mathrm{J}_{\text {leak }}$ (green curve), $\mathrm{J}_{\mathrm{SRH}}$ (orange curve), $\mathrm{J}_{\text {Auger }}$ (pink curve), $\mathrm{J}_{\text {rad }}$ (blue line) and $\mathrm{J}_{\text {tot }}$ (black curve) are also presented.

It is evident that for $\mathrm{T}<40 \mathrm{~K}$, the contributions from all three non-radiative mechanisms were negligible compared with the experimental data $\left(\mathrm{J}_{\exp }\right)$, thus the high $\mathrm{J}_{\text {th }}$ in the low $\mathrm{T}$ region can only be attributed to radiative recombination. The $\mathrm{J}_{\mathrm{rad}}$ was fixed at $2.5 \mathrm{kA} / \mathrm{cm}^{2}$ to make a reasonable fit between $\mathrm{J}_{\text {tot }}$ and $\mathrm{J}_{\exp }$. This plot also implies that $\mathrm{J}_{\text {leak }}$ is the dominant non-radiative component above $50 \mathrm{~K}$. In fact, according to this calculation, at $120 \mathrm{~K}$ only $17 \%$ of the total current contributed towards radiative recombination, while leakage current consumed $67 \%$ of the total current. Within $\mathrm{J}_{\text {leak, }}$, the hole leakage was the dominating factor, since there was no valence band offset between the waveguide and $\mathrm{n}$-cladding. The threshold current density at $4 \mathrm{~K}$ is rather high and requires explanation. According to Figure 8, the main contribution to the threshold current at this temperature is radiative. Because there is a distribution of QD size and composition, only a small fraction of QDs achieve threshold at the same injection level, and a large part of the radiative current is essentially wasted. Figure 4 confirms that QD of different sizes give coherent emission at different injection levels, with the larger QD being the first to reach threshold. We can speculate that only $~ 1 \%-10 \%$ of the QD are lasing together at each temperature.

\section{Conclusions}

We investigated the gain and characteristic temperature dependence of type II In(As)Sb/InAs QDs for use in mid-infrared laser diodes. The devices emitted at around $3.1 \mu \mathrm{m}$ and operated up to $120 \mathrm{~K}$. The transition energy dependences on QD size and composition were calculated. The charging effect 
in the QDs was identified as the main reason for the blue shift in the spontaneous electro-luminescence. Different non-radiative recombination mechanisms were compared, and it was found that the leakage current was the major factor for the increase of $\mathrm{J}_{\mathrm{th}}$ with temperature, though the $\mathrm{J}_{\mathrm{rad}}$ needed to obtain lasing threshold was also quite high. In order to improve the $\mathrm{T}_{\max }$ and reduce $\mathrm{J}_{\text {th }}$, the $J_{\text {leak }}$ should be effectively suppressed, probably by inserting a hole blocking layer in the laser structure. The $\mathrm{J}_{\text {rad }}$ also needs to be reduced, which can only be achieved by increasing the modal gain of the QDs and carefully controlling the size distribution of the QDs.

\section{Acknowledgments}

This work was supported by the European Marie Curie Initial Training Network PROPHET, Grant No. 264687. We are grateful to Min Yin and Jonathan Hayton for the LPE growth of the InAs0.61 $\mathrm{Sb}_{0.13} \mathrm{P}_{0.26}$ cladding layers.

\section{Author Contributions}

Qiandong Zhuang and Qi Lu did the MBE growth of the laser structure. Qi Lu fabricated the laser diodes, characterized the devices, analyzed the performances and wrote the manuscript. Anthony Krier supervised the work and modified the article.

\section{Conflict of Interest}

The authors declare no conflict of interest.

\section{References}

1. Krier, A.; Yin, M.; Smirnov, V.; Batty, P.; Carrington, P.J.; Solovev, V.; Sherstnev, V. The development of room temperature LEDs and lasers for the mid-infrared spectral range. Phys. Status Solidi(a) 2008, 205, 129-143.

2. Yin, Z; Tang, X. A review of energy bandgap engineering in III-V semiconductor alloys for mid-infrared laser applications. Solid. State. Electron. 2007, 51, 6-15.

3. Range, W.; Hosoda, T.; Kipshidze, G.; Tsvid, G.; Shterengas, L.; Belenky, G. Type-I GaSb-based laser diodes operating in 3.1- to 3.3-um wavelength range. IEEE Photon. Technol. Lett. 2010, 22, 718-720.

4. Yao, Y.; Hoffman, A.J.; Gmachl, C.F. Mid-infrared quantum cascade lasers. Nat. Photonics 2012, 6, 432-439.

5. Vurgaftman, I.; Bewley, W.W.; Canedy, C.L.; Kim, C.S.; Kim, M.; Lindle, J.R.; Merritt, C.D.; Abell, J.; Meyer, J.R. Mid-IR type-II interband cascade lasers. IEEE J. Sel. Top. Quant. Electron. 2011, 17, 1435-1444.

6. Coleman, J.J.; Young, J.D.; Garg, A. Semiconductor quantum dot lasers: a tutorial. Semicond. Sci. Technol. 2011, 29, 499-510.

7. Solov'ev, V.A.; Carrington, P.; Zhuang, Q.; Lai, K.T.; Haywood, S.K.; Ivanov, S.V.; Krier, A. $\mathrm{InSb} / \mathrm{InAs}$ nanostructures grown by molecular beam epitaxy using $\mathrm{Sb}_{2}$ and $\mathrm{As}_{2}$ fluxes. In 
Proceedings of the 13th International Conference on Narrow Gap Semiconductors 2007, Guildford, UK, 8-12 July 2007; pp. 129-131.

8. Carrington, P.J.; Solov'ev, V.A.; Zhuang, Q.; Krier, A.; Ivanov, S.V. Room temperature midinfrared electroluminescence from InSb/InAs quantum dot light emitting diodes. Appl. Phys. Lett. 2008, 93, 091101.

9. Carrington, P.J.; Solov'ev, V.A.; Zhuang, Q.; Ivanov, S.V.; Krier; A. InSb quantum dot LEDs grown by molecular beam epitaxy for mid-infrared applications. Microelectron. J. 2009, 40, 469-472.

10. Solov'ev, V.A.; Sedova, I.V.; Lyublinskaya, O.G.; Semenov, A.N.; Mel’tser, B.Y.; Sorokin, S.V.; Terent, Y.V.; Ivanov, S.V. Midinfrared injection-pumped laser based on a III-V/II-VI hybrid heterostructure with submonolayer InSb insets. Tech. Phys. Lett. 2005, 31, 235-237.

11. Yamaguchi, K.; Yujobo, K.; Kaizu T. Stranski-Krastanov growth of InAs quantum dots with narrow size distribution. Jpn. J. Appl. Phys. 2000, 39, 1245-1248.

12. Wilk, A.; El Gazouli, M.; El Skouri, M.; Christol, P.; Grech, P. Type-II InAsSb/InAs strained quantum-well laser diodes emitting at $3.5 \mu \mathrm{m}$. Appl. Phys. Lett. 2000, 77, 2298.

13. Kirstaedter, N.; Schmidt, O.G.; Ledentsov, N.N.; Bimberg, D.; Ustinov, V.M.; Egorov, A.Y.; Zhukov, A.E.; Maximov, M.V.; Kop'ev, P.S.; Alferov, Z.I. Gain and differential gain of single layer InAs/GaAs quantum dot injection lasers. Appl. Phys. Lett. 1996, 69, 1226.

14. Lelarge, F.; Rousseau, B.; Dagens, B.; Poingt, F.; Pommereau, F.; Accard, A. Room temperature continuous-wave operation of buried ridge stripe lasers using InAs-InP (100) quantum dots as active core. IEEE Photon. Technol. Lett. 2005, 17, 1369-1371.

15. Lu, Q.; Zhuang, Q.; Marshall, A.; Kesaria, M.; Beanland, R.; Krier, A. InSb quantum dots for the mid-infrared spectral range grown on GaAs substrates using metamorphic InAs buffer layers. Semicond. Sci. Technol. 2014, 29, 075011.

16. Yeap, G.H.; Rybchenko, S.I.; Itskevich, I.E.; Haywood, S.K. Type-II InAs ${ }_{x} S_{b_{1-x}} /$ InAs quantum dots for midinfrared applications: Effect of morphology and composition on electronic and optical properties. Phys. Rev. B 2009, 79, 075305.

17. Lu, Q.; Zhuang, Q.; Hayton, J.; Yin, M.; Krier, A. Gain and tuning characteristics of mid-infrared InSb quantum dot diode lasers. Appl. Phys. Lett. 2014, 105, 031115.

18. Joullié, A.; Christol, P. GaSb-based mid-infrared 2-5 $\mu \mathrm{m}$ laser diodes. CR Phys. 2003, 4, 621-637.

19. Janssens, K.L.; Partoens, B.; Peeters, F.M. Magnetoexcitons in planar type-II quantum dots in perpendicular magnetic field. Phys. Rev. B 2001, 64, 155324.

20. Lyublinskaya, O.G.; Solov'ev, V.A.; Semenov, A.N.; Meltser, B.Y.; Terent'ev, Y.V.; Prokopova, L.A.; Toropov, A.A.; Sitnikova, A.A.; Rykhova, O.V.; Ivanov, S.V.; et al. Temperature-dependent photoluminescence from type-II InSb/InAs quantum dots. J. Appl. Phys. 2006, 99, 093517.

21. Ledentsov, N.N.; Bohrer, J.; Beer, M.; Heinrichsdorff, F.; Grundmann, M.; Bimberg, D. Radiative states in type-II GaSb/GaAs quantum wells. Phys. Rev. B 1995, 52, 58-66.

22. Sun, C.; Wang, G.; Bowers, J.E.; Brar, B.; Blank, H. Optical investigations of the dynamic behavior of GaSb/GaAs quantum dots. Appl. Phys. Lett. 1996, 68, 1543-1545. 
23. Gradkowski, K.; Pavarelli, N.; Ochalski, T.J.; Williams, D.P.; Tatebayashi, J.; Huyet, G.; O'Reilly, E.P.; Huffaker, D.L. Complex emission dynamics of type-II GaSb/GaAs quantum dots. Appl. Phys. Lett. 2009, 95, 061102.

24. Tatebayashi, J.; Khoshakhlagh, A.; Huang, S.H.; Balakrishnan, G.; Dawson, L.R.; Huffaker, D.L.; Bussian, D.A.; Htoon, H.; Klimov, V. Lasing characteristics of GaSb/GaAs self-assembled quantum dots embedded in an InGaAs quantum well. Appl. Phys. Lett. 2007, 90, 261115.

25. Choulis, S.A.; Andreev, A.; Merrick, M.; Adams, A.R.; Murdin, B.N.; Krier, A.; Sherstnev, V.V. High-pressure measurements of mid-infrared electroluminescence from InAs light-emitting diodes at $3.3 \mu \mathrm{m}$. Appl. Phys. Lett. 2003, 82, 1149.

26. Mani, H. Realization and study of a double hetero-structure InAsSb/InAsSbP laser emitting beyond 3 micrometer. (In French) Ph.D. thesis, Université des sciences et techniques de Montpellier 2: Montpellier, France, 1989.

27. Fathpour, S.; Mi, Z.; Bhattacharya, P.; Kovsh, A.R.; Mikhrin, S.S.; Krestnikov, I.L.; Kozhukhov, A.V.; Ledentsov, N.N. The role of Auger recombination in the temperaturedependent output characteristics $(\mathrm{T} 0=\infty)$ of p-doped $1.3 \mu \mathrm{m}$ quantum dot lasers. Appl. Phys. Lett. 2004, 85, 5164-5166.

28. Meyer, J.R.; Felix, C.L.; Bewley, W.W.; Vurgaftman, I.; Aifer, E.H.; Olafsen, L.J.; Lindle, J.R.; Hoffman, C.A.; Yang, M.-J.; Bennett, B.R.; et al. Auger coefficients in type-II InAs/Ga1-xInxSb quantum wells. Appl. Phys. Lett. 1998, 73, 2857-2859.

(C) 2015 by the authors; licensee MDPI, Basel, Switzerland. This article is an open access article distributed under the terms and conditions of the Creative Commons Attribution license (http://creativecommons.org/licenses/by/4.0/). 